\title{
A high performance cost-effective digital complex correlator for an X-band polarimetry survey
}

\author{
Miguel Bergano ${ }^{1 *}$, Armando Rocha ${ }^{2}$, Luís Cupido ${ }^{3}$, Domingos Barbosa ${ }^{4}$, Thyrso Villela ${ }^{5}$, José Vilas Boas ${ }^{5}$, \\ Graça Rocha ${ }^{6,7}$ and George F. Smoot ${ }^{8,9}$
}

*Correspondence: jbergano@av.it.pt ${ }^{1}$ Department of Electronics, Telecommunication and Informatics (DETI), Instituto de Telecomunicações, University of Aveiro, Campus Universitário de Santiago, 3810-193 Aveiro, Portugal Full list of author information is available at the end of the article

\begin{abstract}
The detailed knowledge of the Milky Way radio emission is important to characterize galactic foregrounds masking extragalactic and cosmological signals. The update of the global sky models describing radio emissions over a very large spectral band requires high sensitivity experiments capable of observing large sky areas with long integration times. Here, we present the design of a new $10 \mathrm{GHz}$ (X-band) polarimeter digital backend to map the polarization components of the galactic synchrotron radiation field of the Northern Hemisphere sky. The design follows the digital processing trends in radio astronomy and implements a large bandwidth $(1 \mathrm{GHz})$ digital complex cross-correlator to extract the Stokes parameters of the incoming synchrotron radiation field. The hardware constraints cover the implemented VLSI hardware description language code and the preliminary results. The implementation is based on the simultaneous digitized acquisition of the Cartesian components of the two linear receiver polarization channels. The design strategy involves a double data rate acquisition of the ADC interleaved parallel bus, and field programmable gate array device programming at the register transfer mode. The digital core of the back-end is capable of processing $32 \mathrm{Gbps}$ and is built around an Altera field programmable gate array clocked at $250 \mathrm{MHz}, 1 \mathrm{GSps}$ analog to digital converters and a clock generator. The control of the field programmable gate array internal signal delays and a convenient use of its phase locked loops provide the timing requirements to achieve the target bandwidths and sensitivity. This solution is convenient for radio astronomy experiments requiring large bandwidth, high functionality, high volume availability and low cost. Of particular interest, this correlator was developed for the Galactic Emission Mapping project and is suitable for large sky area polarization continuum surveys. The solutions may also be adapted to be used at signal processing subsystem levels for large projects like the square kilometer array testbeds.
\end{abstract}

Keywords: Radioastronomy (astronomy, astrophysics and cosmology), Logic design (hardware), Register-transfer-level implementation (hardware)

\section{Background}

The plethora of radio and microwave experiments exploring large radio spectral bands from the Megahertz to many dozens of Gigahertz promise a very high science impact on galactic and extragalactic radio astronomy (RA). The concurring radio surveys planned 
with many instruments, including those to be performed as parts of Key Science projects of the square kilometer array (SKA) and its pathfinders and precursors require the refining of the radio global sky models (GSM) and knowledge improvement of galactic foreground radiation. This improvement enables a better separation of the different emission critical components to the analysis of the cosmic microwave background (CMB) observations obtained by ground and space missions above $70 \mathrm{GHz}$ (Komatsu et al. 2011; Planck Collaboration 2013). Furthermore, it allows a better separation of low frequency components below $1.4 \mathrm{GHz}$ from observations obtained with radio-interferometers like the SKA and its pathfinders precursors (LOFAR, MWA, MeerKAT, ASKAP). The necessary high sensitivity large scale mapping in radio requires long observations over large sky areas with exquisite control of receptors systematics. The recent advances in digital radio technologies have raised receiver design to a new level enabling much better systematics control and information processing. Sampling at radio-frequency (RF) and replacing RF and intermediate frequency (IF) analog hardware by digital component subsystems, improves the performance and robustness making it immune to RFI (Johnson et al. 2006). Nowadays, digital converters with rates above 1 GSps and field programmable logic devices, capable of parallel digital signal processing at high rates, are widely available and affordable. However, reduction of size, cost and hardware complexity are an advantage at the expense of a more demanding digital design.

The use of digital solutions for applications such as radiometry and polarimetry, carries additional advantages over analog designs. Stability and lower gain fluctuations are advantages that offer more reliability and easier calibration of instruments (Gaier et al. 2012; Gasiewski and Kunkee 1993).

Historically, in 1961 Weinreb (Weinreb 1961) proposed the first single bit radiometer based on a 1 bit digital converters (ADC), a tapped single bit shift register as a delay line and an exclusive or as a multiplier. The solution offered enough sensitivity (64\% of a perfect analog correlator) and paved the way for much of the developments of digital correlator implementations (Bischoff et al. 2013; Cleary 2010; Padin et al. 1993; Piepmeier and Gasiewski 2001). More recently, the collaboration for astronomy signal processing and electronic research (CASPER) and the UNIBOARD project (Parsons et al. 2006; Szomoru 2011; Werthimer 2011) represent an evolution of digital technology applied to radio astronomy. The UNIBOARD uses a complex and expensive system with several Altera Stratix IV FPGAs connected by high-speed links, with a $4 \mathrm{GHz}$ input bandwidth providing 64 output bands. Less demanding applications may however suffer from the CASPER or UNIBOARD solution complexity and its higher cost. A recent paper (Holler et al. 2012) compares the cost of a specific implementation of an analog correlator with a digital equivalent one using CASPER developed boards.

Correlation receivers and polarimeters are extensively described in (Rohlfs and Wilson 2004). We apply it to the development of a new $10 \mathrm{GHz}$ polarimeter, with $1 \mathrm{GHz}$ bandwidth, hence we achieve five times more bandwidth for large sky area polarimetry surveys. This solution uses standards ADCs and an Altera Cyclone III FPGA providing a very cost-effective performance. 


\section{Design and method}

\section{Polarimeter back-end application scenario}

The radio mapping of large sky areas, in the frequency range of $50 \mathrm{MHz}$ to $1 \mathrm{GHz}$, is important to update the GSM and offer a database of legacy templates for future astrophysical use. The mapping of the polarized emission between 5 and $10 \mathrm{GHz}$ projects is the target of several projects such as the Galactic Emission Mapping project (GEM) (Torres et al. 1996) and the more recent C-Band All Sky Survey (CBASS) project (King et al. 2010) to improve the polarized foreground subtraction obtained from CMB missions. The GEM project began to map the polarized microwave emission of the sky in Brazil, having a counterpart in the Northern Hemisphere, after the installation of an antenna in Portugal (Fonseca et al. 2006). This combination of observations will cover about $85 \%$ of the sky.

Figure 1 represents an illustration of the Northern hemisphere scanning strategy and the $10 \mathrm{GHz}$ all-sky template obtained after extrapolation of GSM templates from (Giardino et al. 2002). The Fiducial values on sky flux for receiver and mapping strategy design were obtained from the simulated sky at $10 \mathrm{GHz}$ and incorporating the radio synchrotron spectral index corrections after (Planck Collaboration et al. 2015; Giardino et al. 2002). Initiated as part of the GEM collaboration and building on the lessons of the C-band (5 GHz) polarimeter (Bergano et al. 2007) here we outline the X-band (10 GHz) new digital design. The foreground removal at lower frequencies requires the measurement of sky noise temperature with a resolution of a few micro Kelvin. To enhance the sensitivity and reduce the sky survey integration time to acceptable values, the bandwidth must be at least $1 \mathrm{GHz}$ or higher.

\section{Polarimeter analog hardware}

The polarimeter sensitivity and dynamic range depend on antenna, system noise and integration time, thus influencing the digital design of the backend, where final detection of Stokes parameters takes place. The expected polarized signal power equivalent temperature at $10 \mathrm{GHz}$ is $\sim 0.1 \mathrm{mK}$ so a long integration time is needed. The antenna is a $9 \mathrm{~m}$ Cassegrain type with an HPBW $\Theta_{3 \mathrm{~dB}}=0.28^{\circ}$ and equipped with a corrugated feed horn. A polarizer followed by an orthomode transducer (OMT) carries the separation between left and right circular polarizations (LHCP and RHCP). Both polarization channels are further amplified until they are digitized. The analog front end block diagram of the receiver for one channel is depicted in Fig. 2. In short, the analog chain is responsible for amplifying the signals to the required digital entry levels. Since the galactic synchrotron emission is linearly polarized, we designed the polarimeter to output the linear $U$ and V Stokes parameters through the correlation of the incoming circular polarization components and thus minimize calibration systematics and output Stokes parameters.

\section{The analog receiver chain}

The signal in Fig. 1 is amplified using a low noise amplifier (LNA) (LNC7-10A from Low Noise Factory) operating at $70 \mathrm{~K}$ inside a Dewar. A low loss $1 \mathrm{GHz}$ bandwidth bandpass 

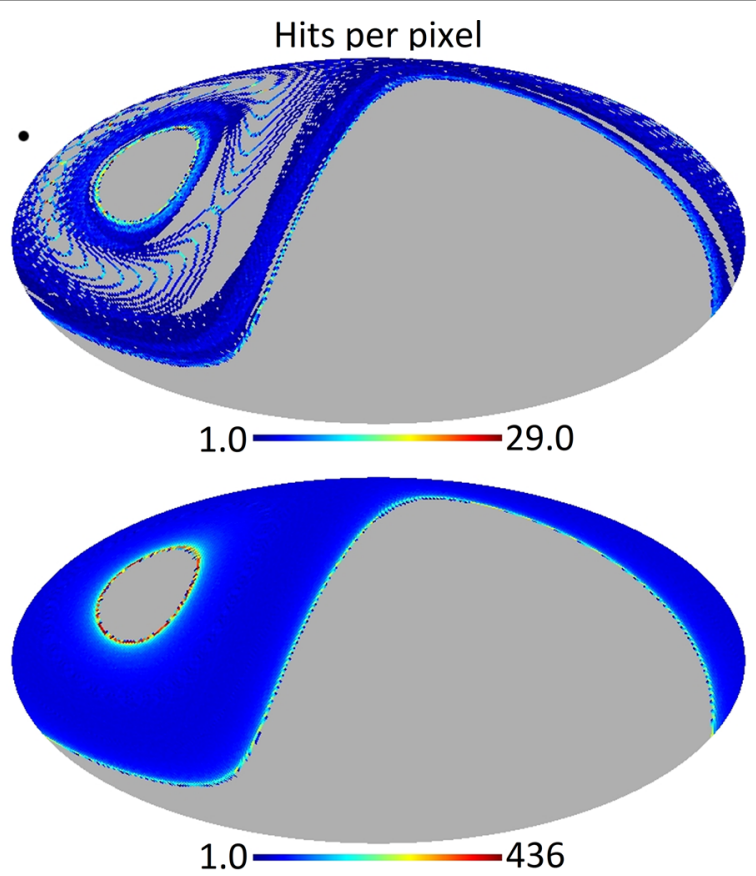

GEM I simulation: $10 \mathrm{GHz}$

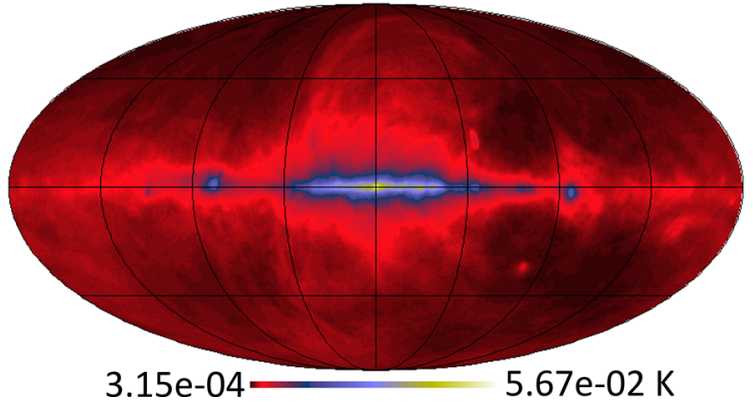

Fig. 1 Simulation of scanning strategy for the Northern GEM survey. From top to bottom simulated $10 \mathrm{GHz}$ total flux, hits/pixel for $1 \mathrm{~h}$ continuous observation; hits/pixel for 1 day continuous observations and hits/pixel for 1 day continuous observations

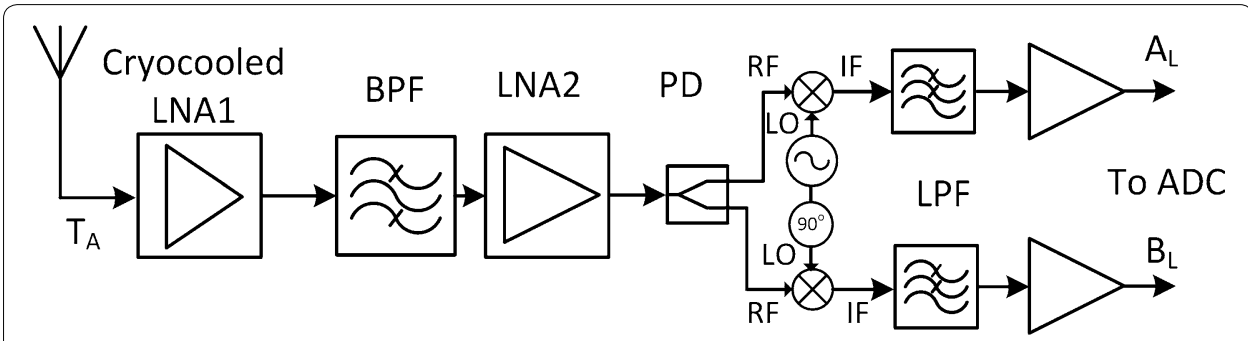

Fig. 2 Front-end block diagram for the left channel depicting the Cartesian components $A_{\llcorner}$and $B_{\perp}$ for the left circular polarization 
waveguide filter labelled (BPF) performs the first stage filtering to remove interferences and reduce the input power at the following amplifying stages. The following amplifying stage labelled (LNA2) uses ultra-low noise pHEMTs and mHEMTs technology devices. Finally, a conversion to baseband, complex in-phase and quadrature components, is performed and followed by $500 \mathrm{MHz}$ bandwidth low-pass filters labelled (LPF) and amplifiers. Each Cartesian component is digitized by one ADC.

The gain/attenuation budget on Table 1 summarizes the relevant system parameters. The system noise temperature $\mathrm{T}_{\mathrm{SYS}}$ includes contributions from the antenna- $\mathrm{T}_{\mathrm{A}}\left(\right.$ for $\mathrm{T}_{\mathrm{A}}$ was assumed a value of $10 \mathrm{~K}$ ), sky- $\mathrm{T}_{\mathrm{SKY}}$ (for $\mathrm{T}_{\mathrm{SKY}}$-atmosphere, spillover, was assumed a value of $5 \mathrm{~K}$ ) and receiver- $\mathrm{T}_{\mathrm{REC}}$ (for $\mathrm{T}_{\mathrm{REC}}$ was assumed a value of $15 \mathrm{~K}$ ) temperatures: $\mathrm{T}_{\mathrm{SYS}}=\mathrm{T}_{\mathrm{A}}+\mathrm{T}_{\mathrm{SKY}}+\mathrm{T}_{\mathrm{REC}}$. The polarimeter design main guidelines take into account the following specifications:

- RF center frequency: $10 \mathrm{GHz}$;

- RF bandwidth B: $1 \mathrm{GHz}$;

- Polarimeter noise temperature $\mathrm{T}_{\mathrm{SYS}}: 30 \mathrm{~K}$;

- Resolution: $\Delta \mathrm{T}=0.1 \mathrm{mK}$ (1st Stokes parameter).

The polarimeter is designed to deliver N estimations (pixels) of the Stokes parameters within the antenna HPBW with $\mathrm{N}=10$ a typical value. Using the antenna HPBW and the revolutions per minute $\omega_{\text {rot }}$ the sky pixel surveying rate, $N_{P}$ (points/s), is given by:

$$
N_{P}=\frac{6 N}{\theta_{3 d B}} \omega_{R o t}
$$

Assuming $\mathrm{N}=10$ and $\omega_{\text {rot }}=1 \mathrm{rpm}$ then $\mathrm{N}_{\mathrm{p}}$ is about 220. The pixel integration time $\mathrm{T}_{\mathrm{p}}$, within the digital backend, is given by:

$$
\tau_{P}=\frac{1}{N_{P}}
$$

and, for the above operating parameters, $\tau_{p}=4.66 \mathrm{~ms}$. The well-known radiometer equation can now be used to estimate the full integration time $\tau$ to achieve the target resolution for the first Stokes parameter (I) at each surveyed pixel:

$$
\tau=\frac{1}{2 B}\left(\frac{T_{S Y S}}{\Delta T}\right)^{2}
$$

Considering $1 \mathrm{GHz}$ bandwidth $\mathrm{B}, \mathrm{T}_{\mathrm{SYS}}=30 \mathrm{~K}$ and $\Delta \mathrm{T}=1 \mathrm{mK}$ the total integration time is $\tau=45 \mathrm{~s}$. The total surveying time $\tau_{\text {Survey }}$ (in days) is given by:

Table 1 Gain/attenuation contributions along the receiver

\begin{tabular}{lcrrrrrrrr}
\hline & T sys & LNA & Filter & 2nd LNA & $\begin{array}{l}\text { Power } \\
\text { splitter }\end{array}$ & Mixer & LPF & Amp. & ADC \\
\hline $\mathrm{T}_{\text {eq }}(\mathrm{K})$ & 30.0 & 3.0 & 438.4 & 20.7 & 288.6 & 1539.8 & 288.6 & 359.2 & \\
NF (dB) & & 0.04 & 4.0 & 0.3 & 3.0 & 8.0 & 3.0 & 3.5 & \\
Gain (dB) & & 35.0 & -4.0 & 15.0 & -3.0 & -8.0 & -3.0 & 52.0 & \\
Power (dBm) & -93.8 & -58.8 & -62.8 & -47.8 & -50.8 & -58.8 & -61.8 & -9.8 & -11.0 \\
\hline
\end{tabular}




$$
\tau_{\text {Survey }}=\frac{\tau}{1440 \tau_{P}}
$$

where 1440 is the number of revolutions per day, so $\tau_{\text {Survey }}$ is about 7 days. The larger the bandwidth $B$ and the smaller the system temperature $T_{S Y S}$ the shorter will be measurement campaign duration.

The calculation of the Stokes parameters follows the work developed in (Bergano et al. 2011).

$$
\begin{aligned}
& I=\left\langle\left(A_{L}+i B_{L}\right) \cdot\left(A_{L}-i B_{L}\right)+\left(A_{R}+i B_{R}\right) \cdot\left(A_{R}-i B_{R}\right)\right\rangle=A_{L}^{2}+B_{L}^{2}-A_{R}^{2}-B_{R}^{2} \\
& Q=\left\langle\left(A_{L}+i B_{L}\right) \cdot\left(A_{L}-i B_{L}\right)-\left(A_{R}+i B_{R}\right) \cdot\left(A_{R}-i B_{R}\right)\right\rangle=A_{L}^{2}+B_{L}^{2}-A_{R}^{2}-B_{R}^{2} \\
& U=2 \operatorname{Re}\left\langle\left(A_{L}-i B_{L}\right) \cdot\left(A_{R}+i B_{R}\right)\right\rangle=2\left(A_{L} A_{R}+B_{L} B_{R}\right) \\
& V=2 \operatorname{Im}\left\langle\left(A_{L}-i B_{L}\right) \cdot\left(A_{R}+i B_{R}\right)\right\rangle=2\left(A_{L} B_{R}-A_{R} B_{L}\right)
\end{aligned}
$$

where $A_{R}, A_{L}, B_{R}$ and $B_{L}$ are the in-phase (A) and quadrature (B) components for the right and left channels complex amplitude and \langle\rangle represents the time average. Analyzing (5) to (8) we can see that a direct implementation requires 8 multipliers and 8 adders. The Stokes parameters resolution depends on the system equivalent noise temperature $\left(\mathrm{T}_{\mathrm{SYS}}\right)$, detection bandwidth (B) and integration time $\left(\tau_{\text {Survey }}\right)$. The first integration is made within the field programmable gate array (FPGA) (Bergano et al. 2011) and the final one by the host acquisition computer.

\section{Digital polarimeter baseline design}

The block diagram of the digital receiver backend is shown in Fig. 3. This diagram begins with a standard FPGA clocked at $250 \mathrm{MHz}$, a clock generator and ADCs with two parallel data buses. It details only, for the sake of simplicity, the acquisition of left channel Cartesian components $\left(\mathrm{A}_{\mathrm{L}}\right.$ and $\left.\mathrm{B}_{\mathrm{L}}\right)$ and the system clock derivation scheme. The required strategies are reached by applying dedicated techniques, namely:

- Two 8 bit ADCs for digitizing each channel Cartesian components (total of four ADCs);

- ADCs with interleaved data buses providing two samples per clock period (presenting 500 MSps per ADC bus to the FPGA);

- Double data rate interfacing to the FPGA, sampling at $500 \mathrm{MSps}$ from the $250 \mathrm{MHz}$ clock.

The ADC achieves 1 GSPS bandwidth by exploiting 1:2 demultiplexing in order to produce two 8 bit streams of samples at half the sample rate. The acquisition of the ADC samples is performed along with its data-clock $(500 \mathrm{MHz})$ divided by two, i.e $250 \mathrm{MHz}$. Figure 3 shows the two ADC streams feeding four shift registers each 8 stages long. Both the rising and the falling edges of the clocks $(250 \mathrm{MHz})$ are used to clock the samples into the proper shift register at the proper time. 


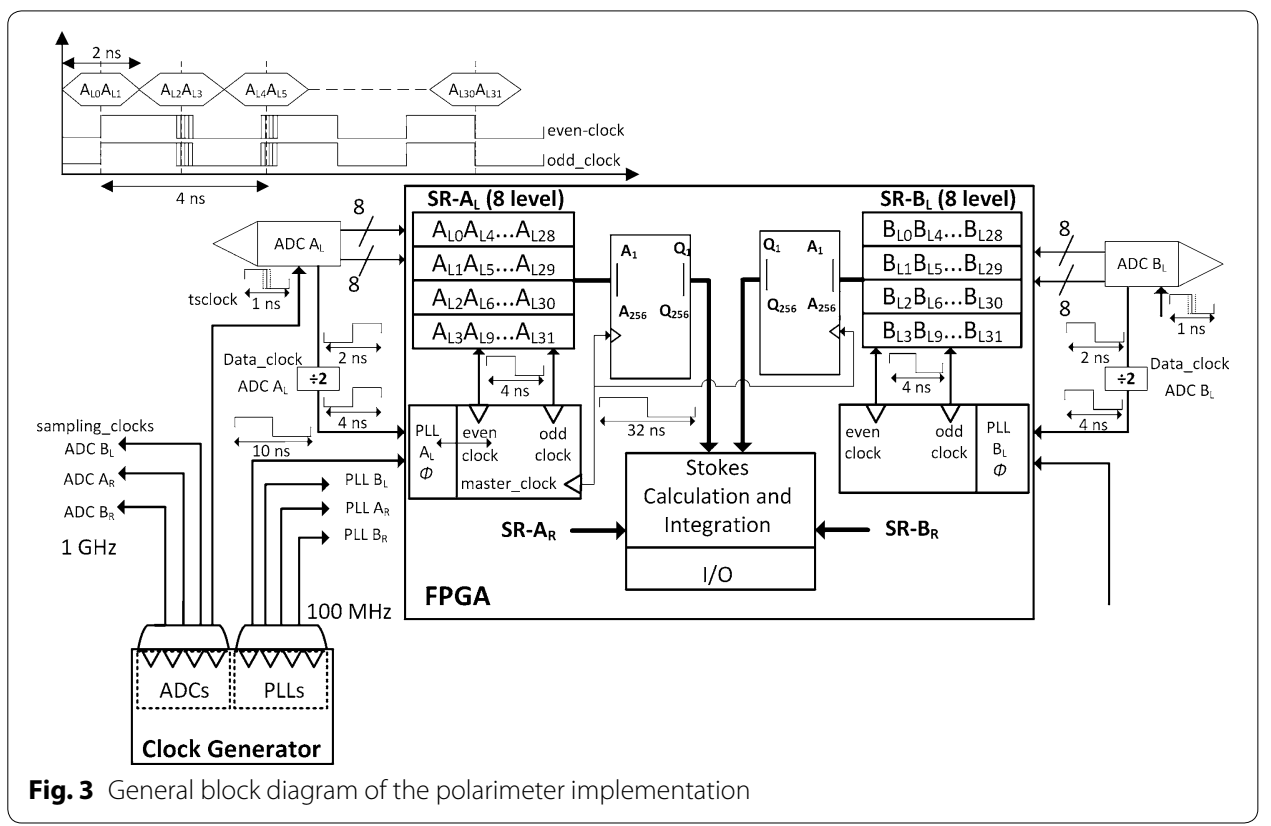

A clock generator chip provides a $1 \mathrm{GHz}$ sampling clock $\left(\mathrm{t}_{\text {Sclock }}\right)$ for each of the four ADCs (ADC $A_{L}$ to ADC $B_{R}$ ). The FPGA phase lock loops (PLL) allow phase adjustable clocks at $250 \mathrm{MHz}$ (even clock and odd clock) that are used to latch the Cartesian component samples to a set of shift registers $\left(S R-A_{L}\right.$ to $\left.S R-B_{R}\right)$ and derives, as well, a 31.25 MHz clock (master_clock) to clock the Stokes parameters calculation blocks. The 8 bit samples $A_{L 0} A_{L 4} \ldots L_{28}$, presented at one of the ADC buses, are latched to the first shift register by the ascending transition of the even_clock (4 ns). The simultaneously available samples $\left(A_{L 1}, A_{L 5} \ldots A_{29}\right)$ at the other $A D C$ bus, are latched to another shift register by the ascending transition of the odd_clock. The samples $A_{L 2} A_{L 6} \ldots A_{30}$ and $\mathrm{A}_{\mathrm{L} 3} \mathrm{~A}_{\mathrm{L} 7} \ldots \mathrm{AL}_{31}$ are stored in the next shift registers with the same strategy but now using the descending transition of the two clocks.

The same solution is implemented to handle the quadrature component of the left channel $B_{L}$ (partially depicted in Fig. 3), as well as the Cartesian components for the right channel $\left(A_{R}\right.$ and $\left.B_{R}\right)$. The four shift registers now hold 32 ns time series of all the receiver Cartesian components sampled at 1 GSps. After 32 samples have been clocked into the shift registers, all 328 bit samples are loaded into a 256-bit register. Further processing will be carried out in blocks clocked by a second clock domain.

The parallel calculation and accumulation of 32 samples of the Stokes parameters (1-4) I, Q, U and V is performed each 32 ns in the Stokes calculation block and integrated in a 22 bits register length. A set of $2 \mathrm{~N}_{\mathrm{S}}$ samples of the integrated Stokes parameters are further integrated in the accumulators (ACC) with 36 bits register length and results are truncated to 24 bits that are enough to resolve $0.1 \mathrm{mK}$ over $30 \mathrm{~K}$ (of $\mathrm{T}_{\text {SYS }}$ ) for the 1st Stokes parameter. Finally, a Multiplexer (MUX)/First In First Out Memory (FIFO) holds the integrated data to be sent to the host computer and performs the data transfer protocol. 
The data transfer rate from the FPGA to the host computer determines the design of the $\mathrm{I} / \mathrm{O}$ hardware and in particular buffer sizes. The data rate $\mathrm{R}_{\mathrm{P}}(\mathrm{bps})$ to transmit all the 4 Stokes parameters (each 3 bytes long) is given by:

$$
R_{P}=96 N_{P}
$$

with $\mathrm{N}_{\mathrm{P}}$ provided by (1). The $21 \mathrm{Kbps}$ transfer rate can be handled by any standard parallel or serial bus.

The delays at ADC bit level, from the FPGA I/O pin to the input shift registers must be well known and equalized and an adequate time alignment of the sampling clock $\left(\mathrm{t}_{\text {Sclock }}\right)$ of the ADC with the FPGA internal latching clock must be achieved. The FPGA must be programmed at a register transfer level to assign the I/O pins and route all the bits to the shift registers with predictable delays. Also, the latching clocks must have an adjustable phase. The digital hardware design requirements are summarized below:

- Reduced time dispersion from I/O pin to input registers;

- ADC located strategically close to the FPGA dedicated PLLs;

- Adequate clock distribution and FPGA PLLs programming.

We now describe the hardware parts selection strategy and perform an analysis of the clock and timing requirements for the proposed solution.

\section{Experimental measurements and results}

The results presented below were obtained from the fabricated and assembled printed circuit board (PCB). The board with its assembled components is about $14 \times 20 \mathrm{~cm}$ and is presented in Fig. 4.

The board has four layers in the following order (from top to bottom): Signal, Ground, Power and Signal. The main chip components observed in the highly symmetrical layout are the FPGA (Cyclone III - EP3C55F780C6), the ADCs (ADC081000) positioned at the

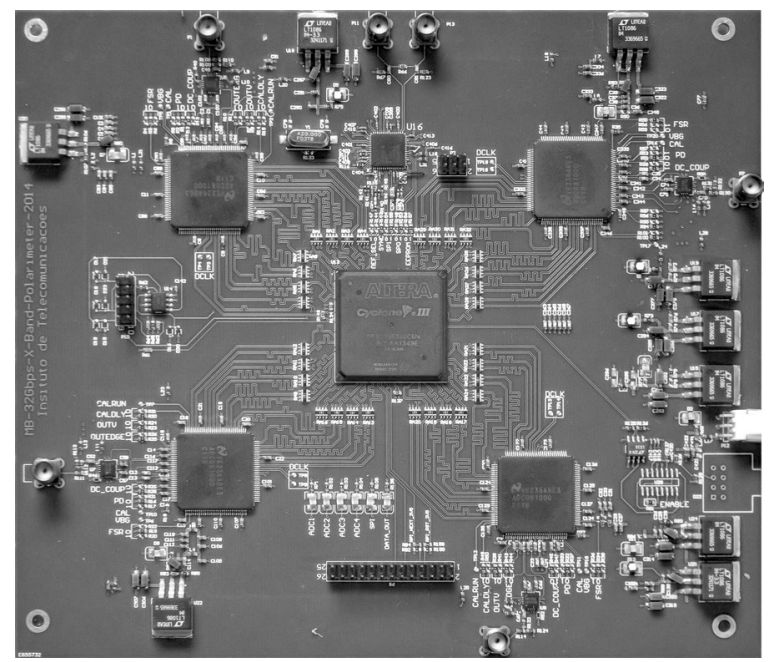

Fig. 4 Correlator printed circuit board 
FPGA corners, the differential ADC drivers (LMH6555) close to the ADC and the clock generator (AD9522-4). Between the two bottom ADCs is the general purpose input output (GPIO) bus connector. At the left side are the configuration devices of the FPGA (Active Serial) and one fiber link (ST connector near the border). At the rightmost side of the layout are the power supply voltage regulators.

The use of single ended FPGA pins requires their physical separation from the differential input pins. Detailed care was taken with the design of differential lines, separation, coupling, length equalization and termination, power supplies decoupling and signal integrity (Cao et al. 2011; Kim et al. 2010; Sharawi 2004).

As long as the connections between elements (I/O pin to register) remain short enough and with small length dispersion to stay within the clock sampling time window, it is possible to implement a standard synchronous pipeline architecture. The pins transmission directly to an input register helps to maximize the timing performance and allows faster setup times.

The reconstruction of several input signal sinusoids with different amplitudes and frequencies were tested. However, we opted to only present in Fig. 5 the reconstruction of an acquired sinusoid with a power of $-10 \mathrm{dBm}$ and $10 \mathrm{MHz}$ frequency.

The previous figure clearly demonstrates an excellent acquisition of the input sinusoids. The small variations of the acquired sinusoid points are due to an improper digitization of the first and/or second least significant bits due to inherent noise of the system.

The second test phase consisted on obtaining the Stokes parameters for the linear, circular and partial polarizations. The operation of the correlator is simulated at the laboratory by inserting several polarization scenarios at the correlator.

The best approach here was to send the Stokes parameters time series, for later analysis, to a small computer using a GPIO interface. Three scenarios were considered: one for linear polarization, one for circular polarization and other for partial polarization. Two sinusoids with different amplitudes and frequencies for each polarization are created to mimic these polarizations Fig. 6. We opted to present the results of a circular polarization situation, that is, two sinusoids with the same amplitude and frequency and phase imbalance of $\pi / 2$ radians. The power of the testing signal was manually switched during a few seconds through the levels of $-12 \mathrm{dBm},-18 \mathrm{dBm},-24 \mathrm{dBm}$ and $-30 \mathrm{dBm}$. The test results are shown in Figs. 7 and in 8.

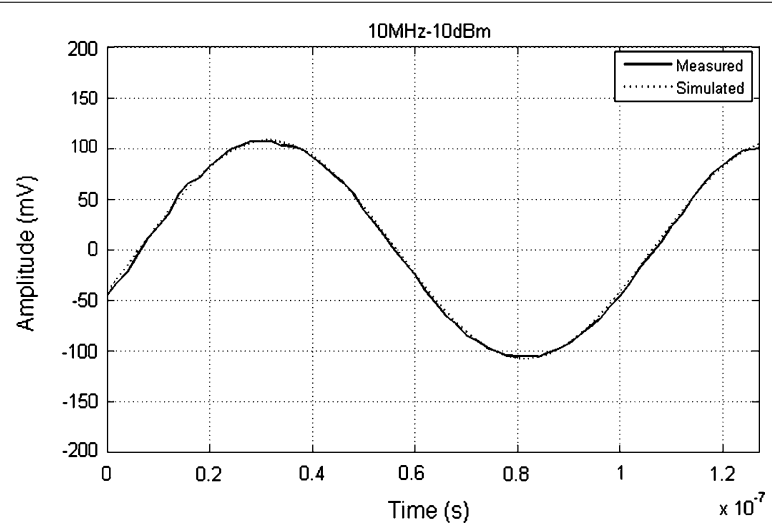

Fig. 5 Acquisition of a sinusoid at $10 \mathrm{MHz}$ : simulated versus acquired time series 


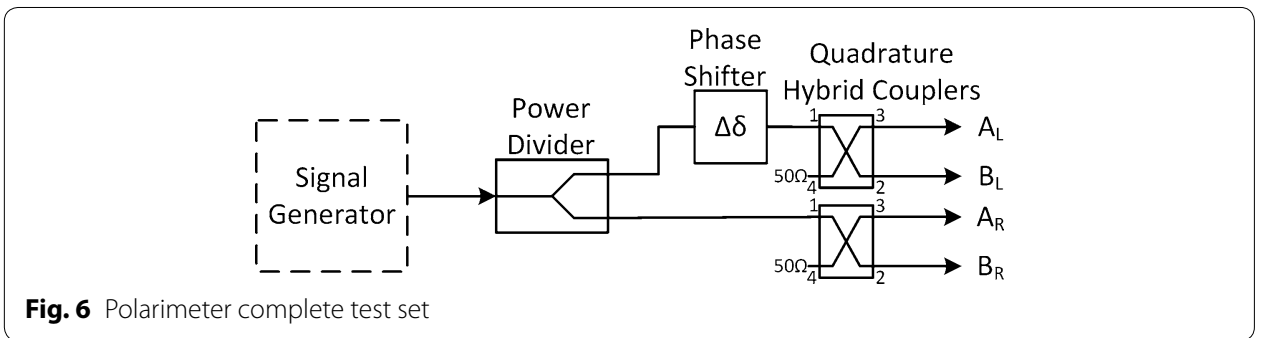

Fig. 6 Polarimeter complete test set

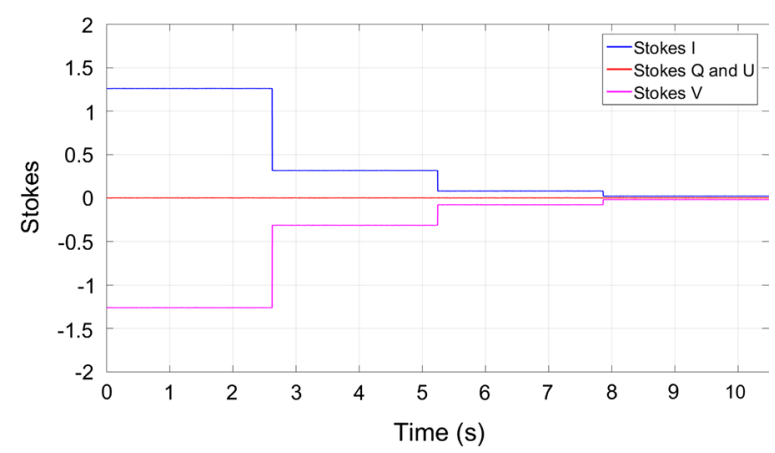

Fig. 7 Linear left polarization Stokes parameters for four amplitude levels

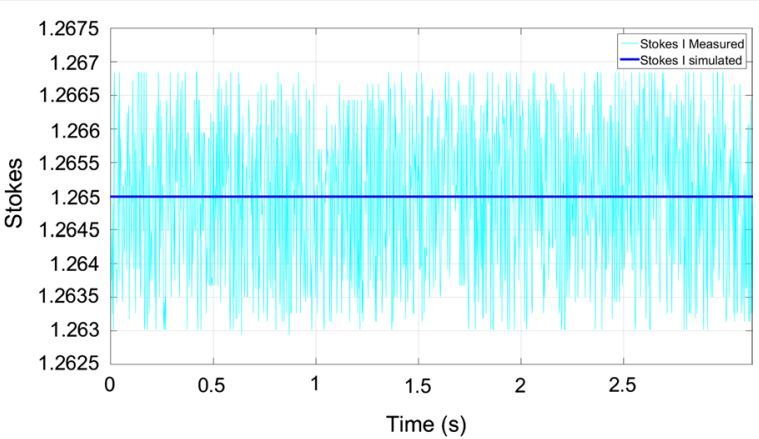

Fig. 8 Linear left polarization Stokes I parameter: simulated versus measured

The time series shown corresponds to the Stokes parameter calculation considering the acquisition of two sinusoidal signals of $10 \mathrm{MHz}$ described above at a rate of $1 \mathrm{GSps}$. The residual differences between the measured and simulated results are in line with the inaccuracy of the simulated amplitude sinusoid which corresponds to a standard deviation of 0.009 , or a SNR of $34 \mathrm{~dB}$.

The test to other polarizations (partial polarization) demonstrated a lower value of the standard deviation (to 0.01 ) proving that a correct operation of the cross correlation Stokes parameters (U and V). The tests consisted on receiving 5000 samples (for each Stokes parameter) from the correlator, each sample represents an average of the 65,536 accumulations performed in the correlator. Since each accumulation takes $2 \mathrm{~s}$ a sole test takes $10 \mathrm{~s}$ as presented in Fig. 7.

The layout of the test bench is presented in Fig. 9.

A summary of the used FPGA resources is presented in Table 2. 


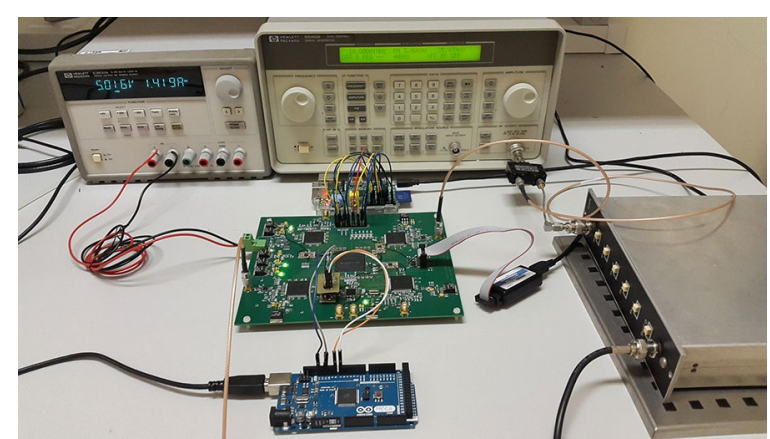

Fig. 9 Layout of the polarimeter test bench

Table 2 Resources used by the FPGA

\begin{tabular}{ll}
\hline Device cyclone III FBGA 780 pins & EP3C55F780C6 \\
\hline Total logic elements & $10,637 / 55,856(19 \%)$ \\
Total combinatory functions & $6340 / 55,856(11 \%)$ \\
Dedicated logic registers & $5273 / 55,856(9 \%)$ \\
Total registers & 5337 \\
Total memory bits & $1032 / 239,6160(<1 \%)$ \\
Embedded multiplier 9-bit elements & $256 / 312(82 \%)$ \\
Total PLLs & $4(100 \%)$ \\
\hline
\end{tabular}

Finally, Table 3 summarizes the hardware price contributions for a prototype. This table confirms the possibility of a low to medium scale production for less than $€ 1000$.

\section{Conclusions}

The design of wide bandwidth digital receivers based on fast ADCs and FPGA data processing is important for RA applications requiring large integration time and where calibration procedures and receiver stability are mandatory. These digital designs require agile interference detection and cancellation, communication systems using spatial diversity and FPGAs smart coding.

The development of a cost effective $1 \mathrm{GHz}$ bandwidth digital polarimeter required implementation of the VLSI Hardware Description Language (VHDL) code at the register transfer level with a high degree of parallelization. The device clock distribution is based on an external clock generator combined with FPGA PLL resources to drive all the system (FPGA and 4 ADCs) with adequate timing and high synchronization.

The achieved results prove the hypothesis of having a low range FPGA as a data processing unit operating at high frequency $(500 \mathrm{MHz})$ without the use of dedicated high

Table 3 Estimated price for the correlator PCB

\begin{tabular}{lc}
\hline $\mathbf{5 4 0}$ components in $\mathbf{4}$ layers & FR4 1.6 $\mathbf{~ m m}$ \\
\hline PCB fabrication & $315 €$ \\
PCB assembly & $305 €$ \\
Components & $755 €$ \\
Total price & $1375 €$ \\
\hline
\end{tabular}


speed buffers [Multi Gigabit Transceiver (MGT), Rocket I/O] or external devices, such as serializers, that increase the price and complexity of the fabrication. The main goal is to operate the ADCs, FPGA and clock generator at its maximum limits. Indeed, this digital backend enables GEM to proceed towards acquisition of data over large sky areas in X-band with high sensitivity. As a corollary, projects with massive needs of digital processing subsystems like the SKA, may benefit from this approach since the main hardware characteristic of this high bandwidth design is the use of a conventional FPGA and off-the-shelf components, making the design affordable for projects requiring costeffective digital processing.

\begin{abstract}
Abbreviations
GSM: global sky model; Gbps: giga bits per second; GSps: giga sample per second; ADC: analogic to digital converter; FPGA: field programmable gate array; GEM: galactic emission mapping; VHDL: VLSI hardware description language; RA: radio astronomy; SKA: square kilometer array; LOFAR: low-frequency array; MWA: murchison widefield array; MeerKAT: Karoo Array Telescope; ASKAP: Australian square kilometer array pathfinder; RF: radio frequency; IF: intermediate frequency; RFI: radio frequency interference; CASPER: collaboration for astronomy signal processing and electronic research; CMB: cosmic microwave background; CBASS: C-band all sky survey; HPBW: half-power beam width; OMT: orthomode transducer; LHCP: left hand circular polarizations; RHCP: right hand circular polarizations; LNA: low noise amplifier; pHEMT: pseudomorphic high electron mobility transistor; mHEMT: metamorphic high electron mobility transistor; LPF: low pass filter; PLL: phase lock loop; MSps: mega samples per second; Kbps: kilo bytes per second; ACC: accumulator; MUX: multiplexer; FIFO: first in first out; LVDS: low voltage differential signaling; BGA: ball grid array; I/O: inputs and outputs; PCB: printed circuit board; GPIO: general purpose input/output; MGT: multi gigabit transceiver.
\end{abstract}

\title{
Authors' contributions
}

MB carried out the research work; AR carried out the supervision of the entire work; LC carried out the consultancy to the work; DB carried out supervision of the work. All authors read and approved the final manuscript.

\section{Authors' information}

Miguel Bergano is an electronic engineer from University of Aveiro, Portugal and got his M.Sc. from University of Aveiro. His interests cover Microwave and Radio Astronomy instrumentation. He was collaborator to several projects like PANORAMA (QREN funded), GEM (FCT) and ENGAGE SKA. He has developed high performance devices and instruments for microwave receivers. Currently he is a Ph.D. Student Researcher at the University of Aveiro, associated to the Radio Astronomy Group (http://grit.av.it.pt/_GRIT) in the Telecommunications Institute at Aveiro, where he currently cosupervises cryogenic low noise amplification research activities at the group, proposing, elaborating and participating in different scientific research projects. Armando Rocha was born in LaGuaira, Venezuela. He graduated from the University of Aveiro, Aveiro, Portugal, in 1984 and received the Ph.D. degree in Electrotechnical Engineering from the same University in 1996. He is Auxiliary Professor at the Department of Electronics, Telecommunication and Informatics (DETI) from 1994. His main research activities, developed in DETI and Instituto de Telecomunicações -Polo de Aveiro, Portugal (ITAveiro), are in tropospheric microwave propagation, MIMO channel modelling, remote sensing and equipment development for propagation measurements using digital radio techniques. He participated in several COST projects as national delegate. From 2009 he has been a collaborator with GRIT (Grupo de Rádio-Astronomia do IT-Aveiro, Portugal). Luís Cupido received a degree in electronic engineering from University of Aveiro, Portugal in 1988 and a Ph.D. in technological physics by Instituto Superior Técnico, Lisboa, Portugal in 1997. Since 2013 he is founder and CEO of LC-Technologies (www.cupidotech.com), a company dedicated to the development of scientific instrumentation. From 1997 to 2012 he held a senior research position at IPFN (Instituto de Plasmas e Fusão Nuclear), Lisboa working on millimeter-wave instruments for plasma physics and nuclear fusion research. From 2003 to present date has been a collaborator of the GRIT: Grupo de Rádio-Astronomia do IT (Instituto de Telecomunicações) Aveiro, Portugal. His research interest includes the development of instrumentation in the millimeter and sub millimeter frequency bands with a particular interest on Radio-Astronomy and Nuclear fusion. Mr. Cupido represents Portugal at CRAF (Committee on Radio Astronomy Frequencies) since 2002 and is president of the national section J (Radio Astronomy) of URSI (Union Radio Science International) since 2003. Domingos Barbosa received a degree in Physics and Applied Mathematics by the University of Porto and a Ph.D. in Astrophysics and Space Techniques by the University of Paris VII in 1997. Currently, he leads the GRIT: Grupo de Rádio-Astronomia do IT (Instituto de Telecomunicações) Aveiro, Portugal.) in the Telecommunications Institute of Aveiro, Portugal and coordinates the national infrastructure proposal ENGAGE SKA, promoting academia and industry participation in the square kilometer array project. He was member of the SKA Science and Engineering Committee (20010-2001). He is/was member of several projects like the PrepSKA (FP7), BIOSTRLING 4 SKA (FP7). He was Marie Curie Fellow at the Department of Physics of Imperial College and a Research Visitor at the Astrophysics Group at the Lawrence Berkeley National Laboratory. His research interests include studies on the cosmic microwave Background, galactic radio foregrounds, radio and millimeter waves instrumentation and Green ICT solutions for radio interferometers. Thyrso Villela is Senior Researcher at the Division of Astrophysics of the National Institute for Space Research (INPE), Brazil. He holds a Doctor of Sciences (1987) and a Master in Astronomy (1982) degrees from University of São Paulo, and a B.Sc. in Physics from the University of Brasilia. He was a visiting scholar at the University of California, Berkeley (1983-1986), where he worked at the Space Sciences Laboratory and Lawrence Berkeley National Laboratory. He was President of the Brazilian Astronomical Society (2000-2002) and Director for Satellite Applications and Development (2008-2012) and was Acting President (01/2012-05/2012) of the Brazilian Space Agency. His main interests are in the field of astrophysics (cosmic 
microwave background radiation, cosmology, extragalactic astrophysics, radio astronomy) and scientific instrumentation (mainly microwave and radio techniques). He has developed experiments to operate on telescopes on the ground, and on board satellite and stratospheric balloons. José Vilas Boas Senior Researcher of the National Institute for Space Research (INPE), Brazil, and Member of the Rapporteur Group on SG7D Studies of the National Telecommunication Agency, Brazil. He graduated in Astronomy from the Federal University of Rio de Janeiro and holds Master and a Ph.D. degree in Astrophysics from the National Institute for Space Research. He was post-doctorate from Harvard University (1993). He is Member of the Advisory Committee of the Space and Atmospheric Sciences at INPE and Member of the Users Committee of the Radio Observatory of Itapetinga (ROI), Atibaia, Brazil. His mains interests cover the area of Astronomy and Astrophysics with an emphasis on the following topics: spectroscopy, masers, interstellar medium and molecular clouds. Graça Rocha is a Physics Nobel laureate. He received a Ph.D. in Physics by MIT. He is a Professor of Physics at the University of California, Berkeley, Senior Scientist at the Lawrence Berkeley National Laboratory, and since 2010, a Professor of Physics at the Paris Diderot University, France. He is also Director of the Institute for The Early Universe at Ewha Womans University of Korea under the Korean Ministry of Education, Science and Technology. Among the many honors, he was awarded the Einstein Medal in 2003, the Oersted Medal in 2009 and the Physics Nobel prize in 2006. His prime interests are Experiments on cosmology and particle physics utilizing the cosmic background radiation as a probe of the early universe. These include work on COBE satellite, and several airborne and ground-based experiments. For his achievements he got NASA Medal for Exceptional Scientific Achievement, the Kilby Award and the Productivity Group Award, Goddard Space Flight Center, NASA. George F. Smoot received a Ph.D. in Physics by the University of Cambridge, Physics Department, MRAO, UK, with Research undertaken in the Radio Astronomy Group of the Cavendish Laboratory. She is Staff Research Scientist at Jet Propulsion Laboratory, USA and Visitor at Caltech, Physics Department, and Observational Cosmology group. Among her experience she was also Staff Scientist at the Infrared Processing and Analysis Center, IPAC, in the California Institute of Technology, Caltech and Leverhulme Postdoctoral Fellow at the Cavendish Astrophysics Group, University of Cambridge, UK. She is a Planck Surveyor Scientist with responsibilities in several Core Areas and Core Working Groups in both the Low Frequency Instrument (LFI) and High Frequency Instrument (HFI) Science Core teams. She was or is also a member of the VSA, BICEP, CBASS experiments. Her Research interests include Cosmic Microwave Background analysis, the development of advanced statistical methods for large space data sets analysis, the astrophysical exploitation of microwave data.

\section{Author details}

1 Department of Electronics, Telecommunication and Informatics (DETI), Instituto de Telecomunicações, University of Aveiro, Campus Universitário de Santiago, 3810-193 Aveiro, Portugal. ${ }^{2}$ Department of Electronics, Telecommunication and Informatics (DETI), University of Aveiro, Campus Universitário de Santiago, 3810-106 Aveiro, Portugal. ${ }^{3}$ LC-Technologies, Aveiro, Portugal. ${ }^{4}$ Instituto de Telecomunicações, Campus Universitário de Santiago, 3810-193 Aveiro, Portugal. 5 Instituto Nacionals de Pesquisas Espaciais (INPE) - Divisão de Astrofísica - DAS, Av. dos Astronautas, 1.758, Jd. Granja, CEP 12227-010 São Jose dos Campos, SP, Brazil. ${ }^{6}$ Jet Propulsion Laboratory, M/S 169-327, 4800 Oak Grove Drive, Pasadena, CA 91109, USA. ${ }^{7}$ Caltech, Cahill Building MS 59-33 1200 E. California, Pasadena, CA 91125-3300, USA. ${ }^{8}$ Lawrence Berkeley National Lab, 1 Cyclotron Road, MS 50-5005, Berkeley, CA 94720, USA. ${ }^{9}$ Université Paris-Diderot APC, Bâtiment Condorcet, 10 rue Alice Domon et Léonie Duquet, 75205 Paris Cedex 13, France.

\section{Acknowledgements}

The corresponding author acknowledge support from the Fundação para a Ciência e a Tecnologia (FCT) the Portuguese national funding agency (SFRH/BD/76615/2011) and by FCT/MEC through national funds and when applicable cofunded by FEDER - PT2020 partnership agreement under the project UID/EEA/50008/2013.

\section{Competing interests}

The authors declare that they have no competing interests.

Received: 30 October 2015 Accepted: 5 April 2016

Published online: 19 April 2016

\section{References}

Bergano M et al (2007) Design of an IF section for a galactic emission mapping experiment. Conftele 2007(1):69-72 Bergano M et al (2011) Digital complex correlator for a C-band polarimetry survey. Exp Astron 30(1):23-37 Bischoff C et al (2013) The Q/U imaging experiment instrument. Astrophys J 768(1):9

Cao, Y, Li J, Hu J, Zheng M, Li B (2011) Design consideration for 10 Gbps signal transmission channel in copper backplane system. In: 2011 international conference on computational science and its applications, pp 215-218

Cleary KA (2010) Coherent polarimeter modules for the QUIET experiment. In: SPIE astronomical telescopes and instrumentation: observational frontiers of astronomy for the new decade, vol 9, pp $77412 \mathrm{H}-77412 \mathrm{H}$

Fonseca R et al (2006) Site evaluation and RFI spectrum measurements in Portugal at the frequency range $0.408-10 \mathrm{GHz}$ for a GEM polarized galactic radio emission experiment. New Astron 11:551-556

Gaier TC, Brown ST, Ruf C, Gross S (2012) Wideband agile digital microwave radiometer. NASA Tech Br. (Nov), pp 9-10

Gasiewski AJ, Kunkee DB (1993) Calibration and applications of polarization-correlating radiometers. IEEE Trans Microw Theory Tech 41(5):767-773

Giardino G et al (2002) Towards a model of full-sky galactic synchrotron intensity and linear polarisation: a re-analysis of the Parkes data. Astron Astrophys 387(1):82-97

Holler CM, Jones ME, Taylor AC, Harris Al, Maas SA (2012) A 2-20-GHz analog lag correlator for radio interferometry. IEEE Trans Instrum Meas 61(8):2253-2261

Johnson JT et al (2006) Airborne radio-frequency interference studies at C-band using a digital receiver. IEEE Trans Geosci Remote Sens 44(7):1974-1984 
Kim N, Ahn H, Wyland C, Anderson R, Wu P (2010) Spiral via structure in a BGA package to mitigate discontinuities in multi-gigabit SERDES system. In: 2010 proceedings 60th electronic components and technology conference (ECTC)

King OG et al (2010) The C-band all-sky survey: instrument design, status, and first-look data. In: Proceedings of SPIE millimeter, submillimeter, and far-infrared detectors and instrumentation for astronomy $\mathrm{V}$, pp 7741:774111-1-7741:774111-10

Komatsu E et al (2011) Seven-year Wilkinson microwave anisotropy probe (WMAP) observations. Astrophys J Suppl Ser 192:18

Padin S et al (1993) High-speed digital correlator for radio astronomy. IEEE Trans Instrum Meas 42(4):793-798

Parsons A, Backer D, Werthimer D, Wright M (2006) A new approach to radio astronomy signal processing: packet switched, FPGA-based, upgradeable, modular hardware and reusable, platform-independent signal processing libraries. In: National radio science meeting, vol 3

Piepmeier JR, Gasiewski AJ (2001) Digital correlation microwave polarimetry: analysis and demonstration. IEEE Trans Geosci Remote Sens 39(11):2392-2410

Planck Collaboration, Ade PAR, Aghanim N, Armitage-Caplan C, Arnaud M, Ashdown M, et al (2013) Planck 2013 results. I. Overview of products and scientific results. Astron Astrophys 571:A1

Planck Collaboration, Adam R, Ade PAR, Aghanim N, Arnaud M, Ashdown M, et al (2015) Planck 2015 results. IX. Diffuse component separation: CMB maps. p 42. arXiv:1502.05956 [astro-ph.CO]

Sharawi MS (2004) Practical issues in high speed PCB design. IEEE Potentials 23:24-27

Szomoru A (2011) The UniBoard: a multi-purpose scalable high-performance computing platform for radio-astronomical applications. In: 2011 30th URSI general assembly and scientific symposium, URSIGASS 2011

Torres S et al (1996) The GEM project: an international collaboration to survey galactic radiation emission. Astrophys Space Sci 240(2):225-234

Weinreb S (1961) Digital radiometer. Proc IEEE 49(6):1099

Werthimer D (2011) The CASPER collaboration for high-performance open source digital radio astronomy instrumentation. In: 2011 XXXth URSI general assembly and scientific symposium, pp 1-4

Rohlfs K, Wilson TL (2004) Tools of radio Astronomy, 2nd edn, vol 35. Springer, Berlin

\section{Submit your manuscript to a SpringerOpen ${ }^{\circ}$ journal and benefit from:}

- Convenient online submission

\section{- Rigorous peer review}

- Immediate publication on acceptance

Open access: articles freely available online

- High visibility within the field

Retaining the copyright to your article

Submit your next manuscript at $\boldsymbol{\nabla}$ springeropen.com 